\title{
MicroRNAs Regulate the Pathogenesis of CVB3-Induced Viral Myocarditis
}

\author{
Qinghua Zhanga ${ }^{\mathrm{a}, \mathrm{b}}$ Zonghui Xiao ${ }^{\mathrm{b}}$ Feng He ${ }^{\mathrm{b}}$ Jizhen Zou ${ }^{\mathrm{c}}$ Sha $\mathrm{Wu}^{\mathrm{c}}$ \\ Zhewei Liu ${ }^{a, b}$ \\ a Graduate School of Peking Union Medical College, and Departments of ${ }^{b}$ Molecular Immunology and ${ }^{c}$ Pathology, \\ Capital Institute of Pediatrics, Beijing, China
}

\section{Key Words}

Coxsackievirus B3 $\cdot$ MicroRNA profiling $\cdot$ Real-time

polymerase chain reaction $\cdot$ Target prediction $\cdot$ mRNA

expression $\cdot$ Regulating role

\section{Abstract}

Aims: To evaluate the role of microRNAs (miRNAs) in the pathogenesis of Coxsackievirus B3 (CVB3)-induced viral myocarditis. Methods: We detected miRNA expression profiling by microarray utilizing a mouse model on day 4 after CVB3 infection. Then we validated differentially expressed miRNAs using real-time polymerase chain reaction (PCR). We predicted target genes using miRNA target prediction databases and assessed them using mRNA microarray and qualitative reverse transcription PCR measurements. By analyzing the target function of differentially expressed miRNAs, we initially explored the regulating role of miRNAs in viral myocarditis. Results: We found five differentially expressed miRNAs that are involved in regulating several important innate immune and antiviral pathways such as the Toll-like receptor signaling pathway, RIG-I-like receptor signaling pathway, NOD-like receptor signaling pathway, cytokinecytokine receptor interaction, MAPK signaling pathway, JAK-STAT signaling pathway, and natural killer cell-mediated

\section{KARGER}

Fax +4161306 1234

E-Mail karger@karger.ch

www.karger.com (c) 2012 S. Karger AG, Basel

$0300-5526 / 13 / 0562-0104 \$ 38.00 / 0$

Accessible online at:

www.karger.com/int cytotoxicity. Conclusion: miRNAs regulate the pathogenesis of viral myocarditis. This study may provide a new perspective and a deeper understanding of the pathogenesis of viral myocarditis that may help with the development of novel therapies against CVB3 infection.

Copyright $\odot 2012$ S. Karger AG, Basel

\section{Introduction}

Coxsackievirus B3 (CVB3) is a member of the genus Enterovirus within the family Picornaviridae. It is an important causative agent of virus-induced acute or chronic heart disease in adults and children. Epidemiological studies indicated that $10-20 \%$ of people carrying symptoms of CVB3-induced acute myocarditis will develop chronic disease and that chronic myocarditis progresses to dilated cardiomyopathy at a frequency of 3.5-8.5 cases per 100,000 persons [1]. There is currently no virus-specific preventive or therapeutic procedure available for daily clinical use. An integrated picture of the pathogenesis of viral myocarditis (VMC) is required before effective therapies can be developed.

MicroRNAs (miRNAs), small RNA (approximately 22-nucleotide) molecules that are expressed endogenously 
in cells, are key posttranscriptional regulators of gene expression in multicellular eukaryotes by binding to the 3'untranslational region of target mRNAs $[2,3]$. miRNAs play key roles in the regulation of diverse physiological or pathological processes [4-7]. Host miRNAs can directly interact with viruses to promote or limit viral replication. For example, liver-specific miR-122 binds to sites in the 5 '-untranslational region of the hepatitis $\mathrm{C}$ virus RNA to facilitate hepatitis $\mathrm{C}$ virus replication [8]. In contrast, $\mathrm{miR}$ 32 restricts the replication of primate foamy virus type 1 in human cells [9]. On the other hand, host miRNAs may also indirectly influence virus replication and/or pathogenicity. For example, the vesicular stomatitis virus can induce miR-146a expression that downregulates type I interferon (IFN) production in host cells to increase replication [10], influenza virus can utilize cellular mRNAs to manipulate cellular gene expression to strengthen its pathogenicity [11], and cellular miRNA expression has been significantly modulated in mouse brains upon Venezuelan equine encephalitis virus [12] or rabies virus infection [13]. However, the involvement of miRNAs during CVB3 infection or pathogenesis is largely unknown.

To determine whether cellular miRNAs play a role in the host response to CVB3 infection, we performed an expression profiling of cellular miRNAs in the hearts of CVB3-infected mice and assessed the potential functions of differentially expressed miRNAs by analyzing the predicted target genes. Further, we performed gene microarray analysis and qualitative real-time polymerase chain reaction (qRT-PCR) to verify the expression levels of the predicted targets of the modulated miRNAs within functional pathways. Functional analysis indicated that these differentially expressed miRNAs are involved in several important immune and antiviral response pathways. This report may provide a new perspective for future indepth studies of VMC pathogenesis.

\section{Methods and Materials}

\section{Animals and Virus Challenge}

Male BALB/c mice (6-7 weeks old) were obtained from the Institute of Laboratory Animal Sciences, Peking Union Medical College. All the animal protocols were approved by the Institutional Animal Care and Use Committee. Mice were housed in microisolator cages and food and water were provided ad libitum. A 12-hour light/dark cycle was maintained. All of the experiments with live virus challenge were carried out at the biosafety level 3 facilities of the Laboratory of Molecular Immunology of the Capital Institute of Pediatrics at Beijing, China.

CVB3 was produced from a full-length cDNA clone and amplified in HeLa cells by transfection as described previously [14].
Virus titer was routinely determined at the beginning of the experiment using plaque assay. HeLa cells were cultured in Dulbecco's modified Eagle's medium (DMEM) supplemented with $10 \%$ fetal bovine serum and $100 \mu \mathrm{g}$ of penicillin-streptomycin/ml (Invitrogen, Carlsbad, Calif., USA). Briefly, mice were infected intraperitoneally with $2.0 \times 10^{5}$ plaque-forming units of CVB3 in 200 $\mu l$ of DMEM. Mice that were mock-infected intraperitoneally with DMEM were included as controls.

Histopathology and Immunohistochemistry for CVB3 Antigen

Following CVB3 infection, animals were sacrificed at 4 days postinfection (p.i.), a time frame that brackets acute virus infection in this model. The hearts were then harvested. Ventricular samples were fixed in $10 \%$ neutral-buffered formalin and processed for histopathology and immunohistochemistry staining to confirm the presence and locale of infection, the occurrence and nature of tissue injury, and the extent of inflammation in the hearts. The remaining portions of the samples were immediately snap-frozen in liquid nitrogen and stored at $-80^{\circ}$ for subsequent RNA extractions.

Immunostaining was performed using rabbit monoclonal antibody against CVB3 (Chemicon/Millipore, Temecula, Calif., USA) using a PV-9000 kit (GBI, SEA, USA). Briefly, 4- $\mu \mathrm{m}$ sections were placed on poly-L-lysine-coated slides, deparaffinized, and hydrated. Endogenous peroxidase activity was blocked using 3\% hydrogen peroxide in methanol for $10 \mathrm{~min}$. Sections were incubated for $2 \mathrm{~h}$ with rabbit monoclonal antibody against CVB3 (1:500) at $37^{\circ}$. Slides were washed with phosphate-buffered saline. Polymer helper was added and the slides were incubated for 20 min. The sections were incubated with polyperoxidase-anti-rabbit IgG for $15 \mathrm{~min}$, stained with diaminobenzidine, and counterstained with Harris hematoxylin.

\section{Enzyme-Linked Immunosorbent Assay}

Serum concentrations of IFN- $\gamma$, interleukin (IL)-6, IL-10, and tumor necrosis factor- $\alpha$ (TNF- $\alpha$ ) were determined using a murine enzyme-linked immunosorbent assay (ELISA) kit (R\&D Systems, Chicago, Ill., USA) according to the manufacturer's instructions.

\section{Total RNA Isolation}

Frozen heart sections were minced over ice and transferred to $1.5-\mathrm{ml}$ microcentrifuge tubes. Total RNA was isolated using TRIzol reagent (Invitrogen) according to the manufacturer's protocol. RNA quantity and quality were measured by a NanoDrop ND-1000. RNA integrity was assessed using standard denaturing agarose gel electrophoresis.

\section{miRNA Microarray Analysis}

Five CVB3-infected and 3 mock-infected mice on day 4 p.i. were randomly selected for miRNA microarray assays using the LNA mercury ${ }^{\mathrm{TM}}$ microarray (Exiqon, Vedbaek, Denmark). Total RNA $(1 \mu \mathrm{g})$ was labeled with an $\mathrm{Hy}^{{ }^{\mathrm{TM}}}$ fluorescent label using the miRCURY' Array Power Labeling Kit (Exiqon). The Hy3-labeled RNA samples were hybridized on the miRCURY LNA Array ( $\mathrm{v}$ 16.0) (Exiqon). After being washed, the slides were scanned using the Axon GenePix 4000B microarray scanner. The scanned images were then imported into GenePix Pro 6.0 software for grid alignment and data extraction. Replicated miRNAs were averaged and miRNAs with intensities $\geq 50$ in all samples were chosen for calculating the median normalization factor. Expressed 
Table 1. Primers for selected genes analyzed using qRT-PCR

\begin{tabular}{|c|c|c|c|c|}
\hline $\begin{array}{l}\text { Gene } \\
\text { symbol }\end{array}$ & Gene title & Forward primer & Reverse primer & $\begin{array}{l}\text { Am- } \\
\text { plicon }\end{array}$ \\
\hline TRAF6 & tumor necrosis factor receptor-associated factor 6 & TTCTTGTGTAAACTGTGCTGTGTC & GCAGTCCAGATCATAATGATTAGG & 145 \\
\hline IRAK1 & interleukin-1 receptor-associated kinase 1 & ACCCAGCCCGACAGAAGATG & ATTTGTCAGAGTGAACAAATCAGC & 174 \\
\hline PDCD4 & programmed cell death protein 4 & TGTGGAGGAATGTTTTCAGGC & GTTCTTGGTAACACCTAAGACTGT & 161 \\
\hline IL12A & interleukin-12 subunit alpha & TGCCTGGCTACTAGAGAGACTTCT & CAAGTCCTCATAGATGCTACCAAG & 108 \\
\hline CCL7 & chemokine (C-C motif) ligand 7 & TGTCCCTGGGAAGCTGTTATC & TCCTAGGTTGGTTTCTGTTCAG & 158 \\
\hline$M A P 2 K 3$ & mitogen-activated protein kinase kinase 3 & GGGAGATCGCTGTGTCTATCG & AGCCAGCATCCATTGTCTTTG & 180 \\
\hline SOCS1 & suppressor of cytokine signaling 1 & GGAGCATGCGCGACAGCC & GGTTGCGTGCTACCATCCTAC & 127 \\
\hline$\beta$-Actin & & GAGACCTTCAACACCCCAGC & ATGTCACGCACGATTTCCC & 263 \\
\hline
\end{tabular}

miRNA data were normalized using the median normalization. After normalization, significantly differentially expressed miRNAs were identified using volcano plot filtering between the two groups from the experiment. The threshold we used to screen up- or downregulated miRNAs was a fold change (FC) $\geq 1.5$ and a $\mathrm{p}$ value $\leq 0.05$.

\section{mRNA Microarray Analysis}

The mRNA microarray was conducted using the same archived samples as those used for miRNA microarray. Doublestranded circular DNA (ds-cDNA) was synthesized from $5 \mu \mathrm{g}$ of total RNA using a SuperScript ds-cDNA Synthesis Kit (Invitrogen) and labeled with Cy3 fluorescent dye using a One-Color DNA Labeling Kit (NimbleGen Systems, Madison, Wisc., USA). Microarrays were hybridized at $42^{\circ}$ for $16-20 \mathrm{~h}$ with $4 \mu \mathrm{g}$ of Cy3labeled ds-cDNA. After being washed, the slides were scanned using the Axon GenePix 4000B microarray scanner. The scanned images were then imported into NimbleScan software (NimbleGen Systems; v 2.5) for grid alignment and expression data analysis. The expression data were normalized using quantile normalization and the Robust Multichip Average algorithm. All gene level files were imported into Agilent GeneSpring GX software (v 11.5.1) for further analysis. Differentially expressed genes were identified using volcano plot filtering. The threshold we used was $\mathrm{FC} \geq 2.0$ and a $\mathrm{p}$ value $\leq 0.05$.

\section{Qualitative Real-Time Polymerase Chain Reaction}

We used qRT-PCR to validate the miRNA and mRNA expression changes. Ten samples for each group were applied to the qRTPCR measurement. The qRT-PCR was performed using an ABI 7900 real-time PCR system. miRNA reverse transcription was performed using a miRcute miRNA First-Strand cDNA Synthesis Kit (Tiangen Biochem, Beijing, China) according to the manufacturer's instructions. RT-PCR was performed using a SYBR FAST qPCR Kit (Kapa Biosystems, Boston, Mass., USA) according to the manufacturer's instructions. The verified miRNAs included mmu-miR-146a (target sequence UGAGAACUGAAUUCCAUGGGUU), mmu-miR-23a (target sequence AUCACAUUGCCAGGGAUUUCC), mmu-miR-21 (target sequence UAGCUUAUCAGACUGAUGUUGA), mmu-miR-374 (target sequence AUAUAAUACAACCUGCUAAGUG), and mmu-miR-29a* (target sequence UAGCACCAUCUGAAAUCGGUUA). All samples were run in triplicate. The data were normalized to a U6 RNA control (U6 F: CTCGCTTCGGCAGCACATATACT; U6 R: AC-
GCTTCACGAATTTGCGTGTC), and relative expression was calculated using the $2^{-\Delta \Delta C t}$ method.

For mRNA qRT-PCR, reverse transcription was performed using a TIANScript First-Strand cDNA Synthesis Kit (Tiangen Biochem) according to manufacturer's instructions. Gene and primer names are shown in table 1 . Real-time PCR was performed using a SYBR FAST qPCR Kit (Kapa Biosystems) according to the manufacturer's instructions. Expression of the gene of interest was normalized to that of $\beta$-actin. The gene expression levels were measured in terms of threshold cycle value using the $2^{-\Delta \Delta C t}$ method.

\section{Target Prediction and Functional Analysis of Differentially}

Expressed miRNAs

Three different miRNA target prediction databases (TargetScan, miRBase, and miRanda) were used to infer the targets of the differentially expressed miRNAs. The intersection of these data sets was considered reliable and important [15-17]. Using these identified target genes, we performed the significant gene ontology (GO) classifications and Kyoto Encyclopedia Genes and Genomes (KEGG) pathways that were overrepresented among these target genes to analyze the miRNA functions. For all functional analyses, Fisher's exact test corrected by the false discovery rate method was used to determine the probability that each biological function assigned to that data set was due to chance alone, with p set at 0.05 .

\section{Statistical Analysis}

For the microarray data analysis, expressed miRNA data were normalized using median normalization. After normalization, significantly differentially expressed miRNAs were identified using volcano plot filtering between the two groups of the experiment. The threshold we used to screen up- or downregulated miRNAs was $F C \geq 1.5$ and a $\mathrm{p}$ value $\leq 0.05$. Expression mRNA data were normalized using quantile normalization and the Robust Multichip Average algorithm. All gene level files were imported into Agilent GeneSpring GX software (v 11.5.1) for further analysis. Differentially expressed genes were identified using volcano plot filtering. The threshold we used was $\mathrm{FC} \geq 2.0$ and a $\mathrm{p}$ value $\leq 0.05$.

To assess the statistical significance of GO and KEGG term representations in the predicted miRNA target gene data set, $p$ values were calculated using Fisher's exact test corrected by the false discovery rate method. A GO or KEGG term was considered to be overrepresented within the predicted target genes if the corrected $\mathrm{p}$ value was $<0.05$. 

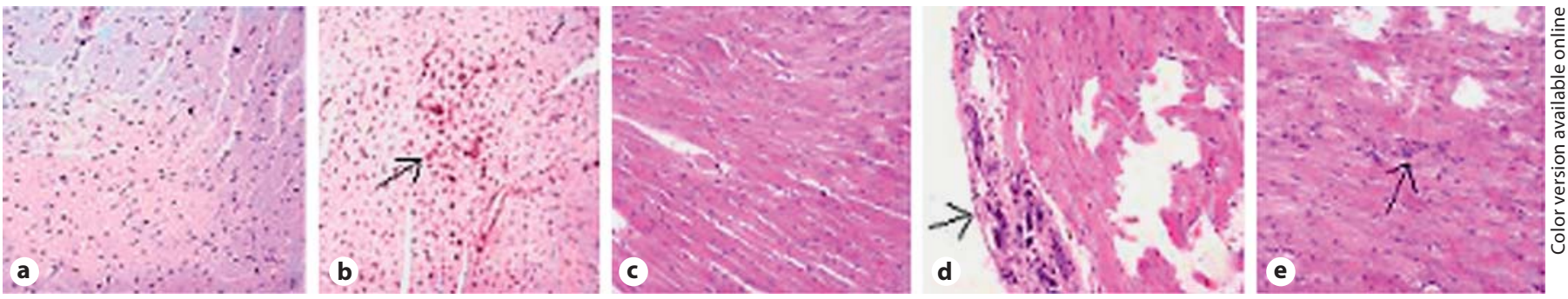

Fig. 1. Confirmation of infection and disease. Mouse hearts on day 4 after CVB3 infection were analyzed using immunohistochemistry (a, b) and hematoxylin and eosin staining (c-e). Immunohistochemistry staining showed CVB3 antigen accumula- tion in myocardial cells (indicated by arrows). Heart tissues have calcification (d) and focal cellular infiltration (e) (indicated by arrows). a, c Mock-infected heart tissues used as a negative control are shown. Magnification $\times 200$.

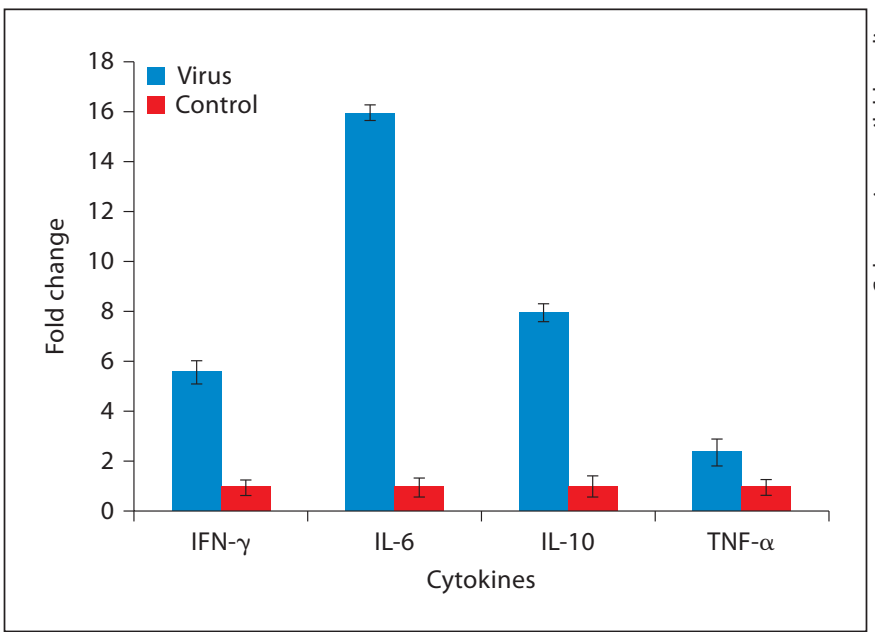

Fig. 2. Cytokine changes in mice infected with CVB3 on day 4 p.i.

\section{Results}

\section{Viral Infection and Disease in the Heart}

To confirm CVB3 infection of the heart muscle, immunostaining for CVB3 antigen in paraffin-embedded heart tissue sections was performed using rabbit monoclonal antibody against CVB3. CVB3 antigen-specific staining showed a greater presence of viral antigen within the myocardial cells indicating CVB3 replication in these cells (fig. 1b). Heart tissues from CVB3-infected mice demonstrated calcification and focal cellular infiltration (fig. 1d, e). Figure 1a and c shows mock-infected heart tissues used as a negative control. We analyzed the day 4 serum cytokine expression levels of IFN- $\gamma$, IL-6, IL-10, and TNF- $\alpha$ and found that their expression levels were clearly increasing (fig. 2).

\section{miRNA Modulation upon CVB3 Infection}

We determined the miRNA expression profile on day 4 p.i. in mouse hearts in response to CVB3 infection. miRNAs whose relative expression levels showed an FC $\geq 1.5$ and $\mathrm{p} \leq 0.05$ were considered significantly upregulated, while those with an FC $\leq 1.5$ and $\mathrm{p} \leq 0.05$ were considered significantly downregulated. As shown in figure 3, the expression levels of five miRNAs were changed, of which four (miR-146a, miR-21, miR-374, and miR-29a*) were found to be significantly upregulated and one (miR-23a) was significantly downregulated. This finding indicated that host miRNAs were modulated in the heart upon CVB3 infection.

\section{Validation of miRNA Expression by $q R T-P C R$}

To validate the differentially regulated miRNAs from the microarray results, we performed a qRT-PCR assessment of the five differentially expressed miRNAs shown in figure 2. The qRT-PCR results of miR-146a, miR-374, miR-21, and miR-29a* were consistent with those of the microarray analysis, but the result of miR-23a was contrary to that of the microarray analysis (fig. 4). The most likely reason for this is that microarray detection is high throughout, whereas RT-PCR has higher sensitivity and specificity.

\section{Target Prediction and Functional Analysis of}

Differentially Expressed miRNAs

For these five differentially expressed miRNAs, miRanda predicted 16,608 target genes, miRBase predicted 3,664 genes, and TargetScan predicted 989 genes. Of these, 146 target genes were predicted by all three systems (fig. 5) and were used for the GO and KEGG pathway analyses. 


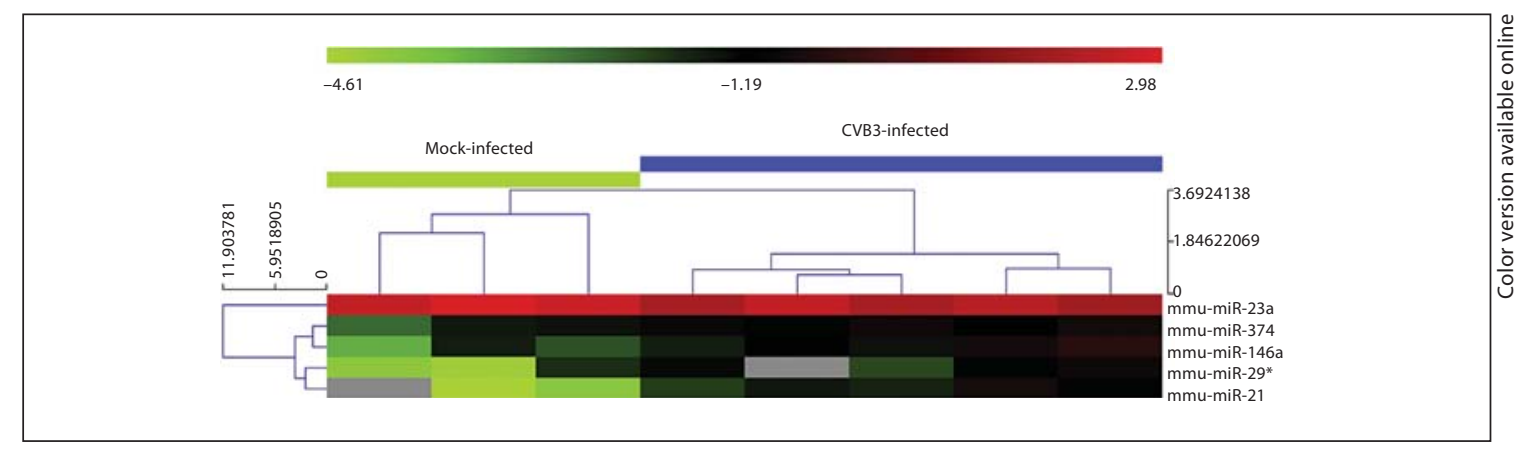

Fig. 3. Heat map and hierarchical clustering of miRNA. The heat map diagram shows the result of the two-way hierarchical clustering of miRNAs and samples ( $\mathrm{p} \leq 0.05$ ). Each row represents an miRNA and each column represents a sample. The miRNA clustering tree is shown on the left and the sample clustering tree is shown at the top. The color scale shown at the top illustrates the relative expression level of miRNA in an individual slide: the red color represents a high relative expression level, while the green color represents a low relative expression level (color only in online version).

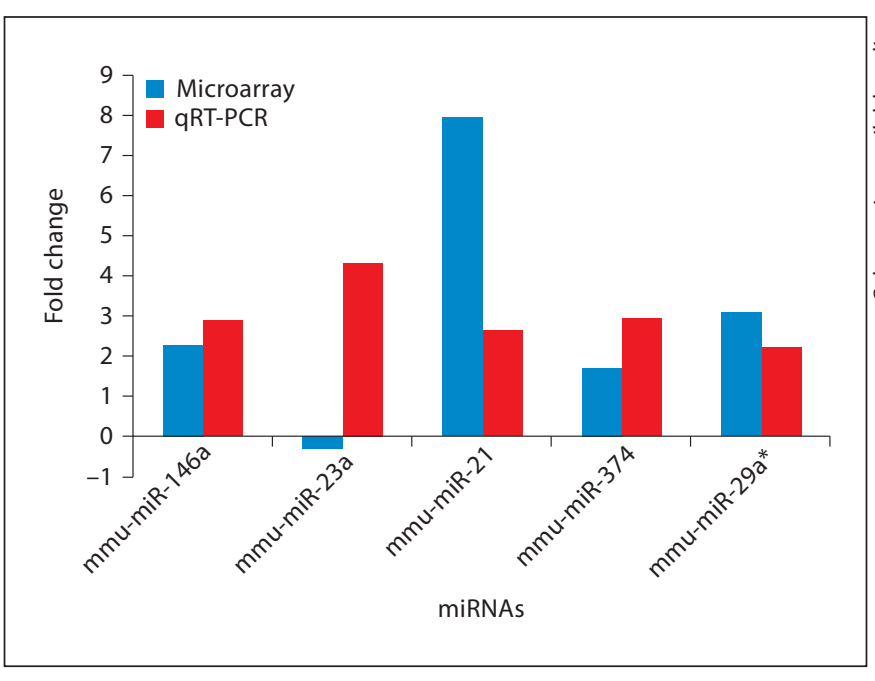

Fig. 4. qRT-PCR confirmation of miRNA microarray findings. Five differentially expressed miRNAs in the hearts infected with CVB3 along with the expression of mock-infected controls were validated using qRT-PCR. The FC of the qRT-PCR was determined using the $2^{-\Delta \Delta C t}$ method and all miRNA expression values were normalized against the U6 endogenous control. Average FC for each miRNA represents that obtained from 10 independent samples.

GO analysis revealed that these predicted targets were involved in 334 biological processes, had 39 molecular functions, and were distributed throughout 33 cellular components $(\mathrm{p}<0.05)$. The ten most common GO categories were cellular process, metabolic process, biological regulation, cellular metabolic process, primary metabolic process, biological process regulation, macromolecule metabolic process, cellular process regulation, cellular macromolecule metabolic process, and metabolic process regulation (fig. 6). These results suggested that CVB3 infection significantly influenced host cellular and metabolic processes in which associated gene expression had been regulated as a result of mRNA microarray analysis (data not shown) and that host cells could be set to regulate these processes to maintain homeostasis.

KEGG pathway analysis revealed 27 statistically remarkable categories $(p<0.05)$. As shown in table 2 , these five differentially expressed miRNAs were found to modulate several important immune-related pathways, such as the Toll-like receptor (TLR) signaling pathway, the RIG-Ilike receptor signaling pathway, the NOD-like receptor signaling pathway, cytokine-cytokine receptor interaction, the MAPK signaling pathway, the JAK-STAT signaling pathway, and natural killer cell-mediated cytotoxicity. Also, one miRNA could modulate many kinds of different signaling pathways, and one signaling pathway could be interchangeably modulated by several miRNAs. These results suggested that miRNAs may effectively modulate the extent of the immune response, the strength, and duration against CVB3 invasion for maintaining host homeostasis.

Together, these results above indicated that miRNAs play a key role in the pathogenesis of CVB3-induced VMC.

\section{mRNA Microarray Assay and qRT-PCR Measurement of miRNA Targets}

miRNAs predominantly function as repressors of target gene expression, and it has recently been shown that their main function in mammalian systems is to decrease target mRNA levels [18]. Therefore, the expres- 
Table 2. Predicted targets of modulated miRNAs upon CVB3 infection involved in the immune response pathways

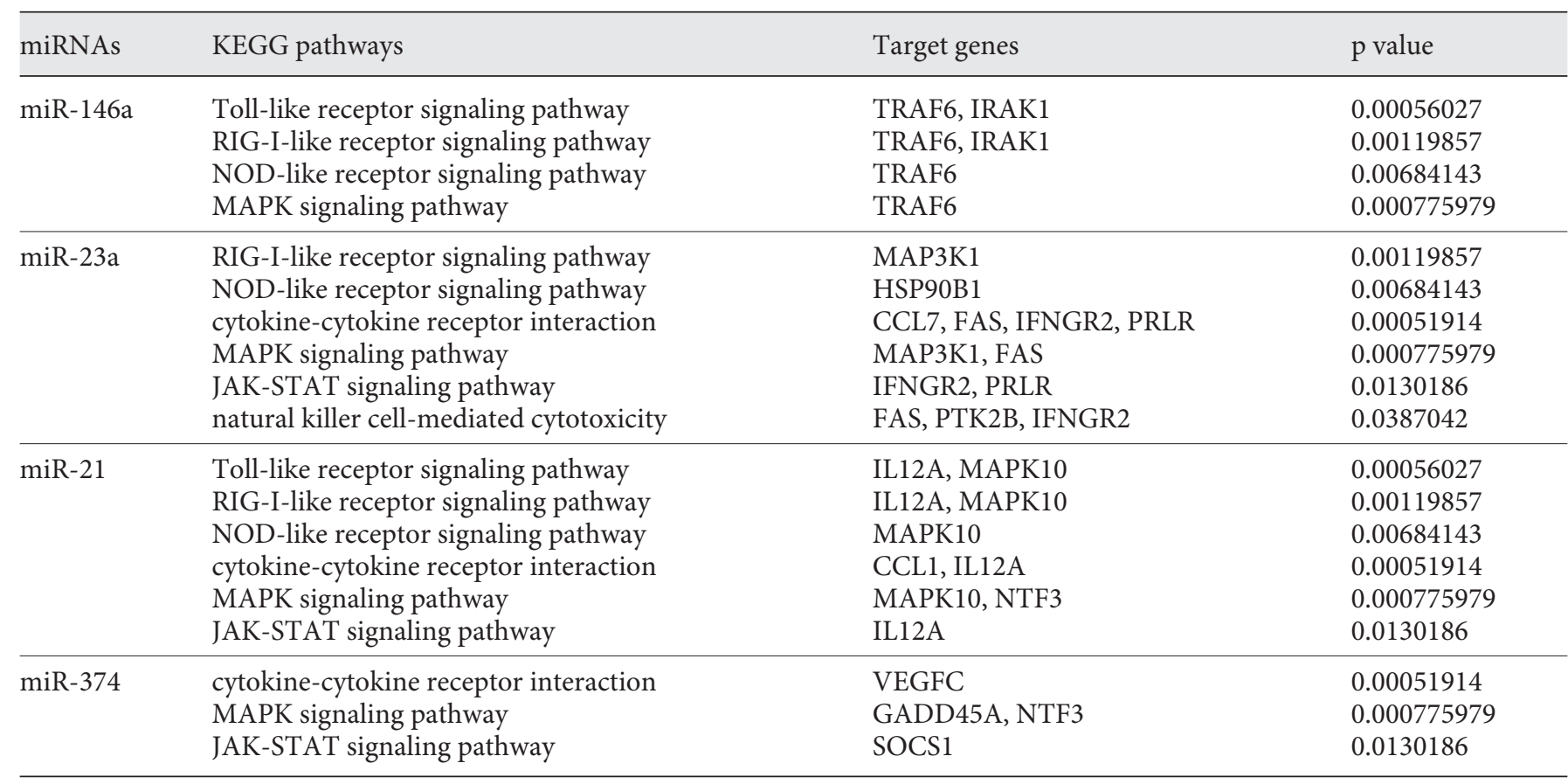

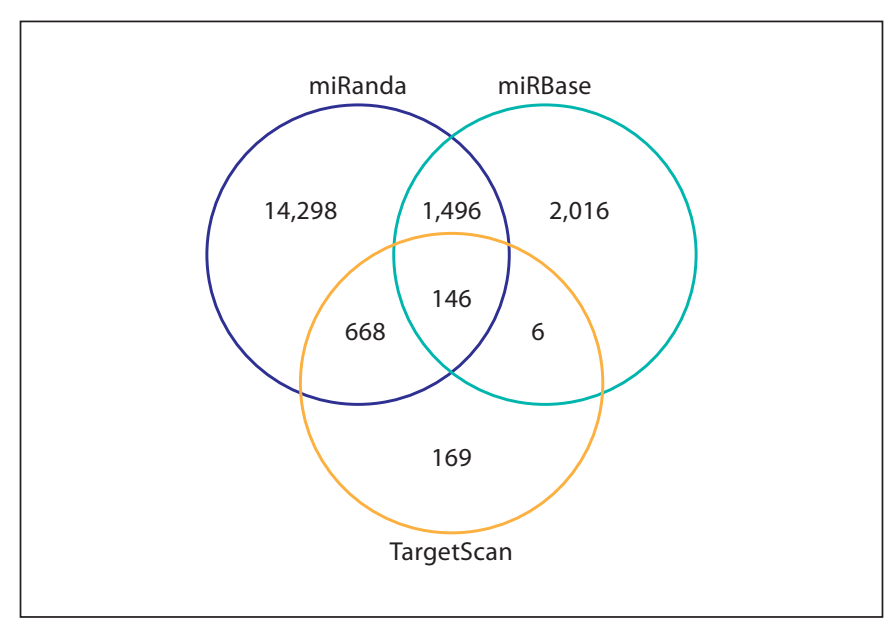

Fig. 5. Overlap of target gene lists predicted for the five miRNAs using the miRanda, miRBase, and TargetScan algorithms.

sion levels of miRNAs and their targets could have a negative correlation. Huang et al. [19] demonstrated that paired expression profiles of miRNAs and mRNAs can be used to identify functional miRNA-target relationships with high precision. As such, we performed an
mRNA microarray assay and a qRT-PCR measurement of miRNA targets. We detected the expression of several genes from the TLR signaling pathway (TRAF6, IRAK1), the RIG-I-receptor signaling pathway (TRAF6, IRAK1), cytokine-cytokine receptor interaction (CCL7, IL12A), the MAPK signaling pathway (MAP2K3), the JAK-STAT signaling pathway (SOCS1), and programmed cell death protein 4 (PDCD4) using qRT-PCR. These targets of the upregulated miRNAs miR-146a, miR-23a, miR-21, and miR-374, respectively, were found to be downregulated (table 3). Together, these results further confirmed that miRNA is involved in CVB3 infection and pathogenesis.

\section{Discussion}

miRNAs comprise an entirely new type of negative regulator of the posttranscriptional regulation of mRNA expression that decreases target protein expression to influence some important biological processes. To date, $>700$ miRNAs have been identified in mammals, and the biological functions of a large portion of them remain elusive. It has recently been determined that miRNA expression profiles have been significantly changed in 
Table 3. Qualitative qRT-PCR analysis of expression of miRNA targets

\begin{tabular}{lllll}
\hline miRNAs & Accession No. & $\begin{array}{l}\text { qRT-PCR } \\
(\text { fold } \uparrow \text { or } \downarrow)\end{array}$ & $\begin{array}{l}\text { Targets of } \\
\text { miRNAs }\end{array}$ & $\begin{array}{l}\text { Accession No. } \\
\text { qRT-PCR } \\
(\text { fold } \uparrow \text { or } \downarrow)\end{array}$ \\
\hline mmu-miR-146a & MIMAT0000158 & $2.90 \pm 0.46 \uparrow$ & TRAF6 & NM_009424 \\
mmu-miR-146a & MIMAT0000158 & $2.90 \pm 0.46 \uparrow$ & IRAK1 & NM_001177973 \\
mmu-miR-23a & MIMAT0017019 & $4.36 \pm 1.39 \uparrow$ & CCL7 & NM_013654 \\
mmu-miR-21 & MIMAT0000530 & $2.62 \pm 0.22 \uparrow$ & IL12A & NM_001159424 \\
mmu-miR-21 & MIMAT0000530 & $2.62 \pm 0.22 \uparrow$ & PDCD4 & NM_001168491 \\
mmu-miR-21 & MIMAT0000530 & $2.62 \pm 0.22 \uparrow$ & MAP2K3 & NM_008928 \\
mmu-miR-374 & MIMAT0003727 & $2.96 \pm 0.55 \uparrow$ & SOCS1 & NM_009896 \\
\hline
\end{tabular}

Data of qRT-PCR are means \pm standard deviation. Average fold change for each miRNA/mRNA represents that obtained from 10 independent samples.

Fig. 6. Enriched GO terms and the number of genes per singular annotation in the biological process category among the differentially expressed miRNAs. The statistical significance was determined by calculating the $p$ value using Fisher's exact test corrected using the false discovery rate method $(p<0.05)$. Each shade of grey represents a different biological process. Only the top ten GO terms are listed here.

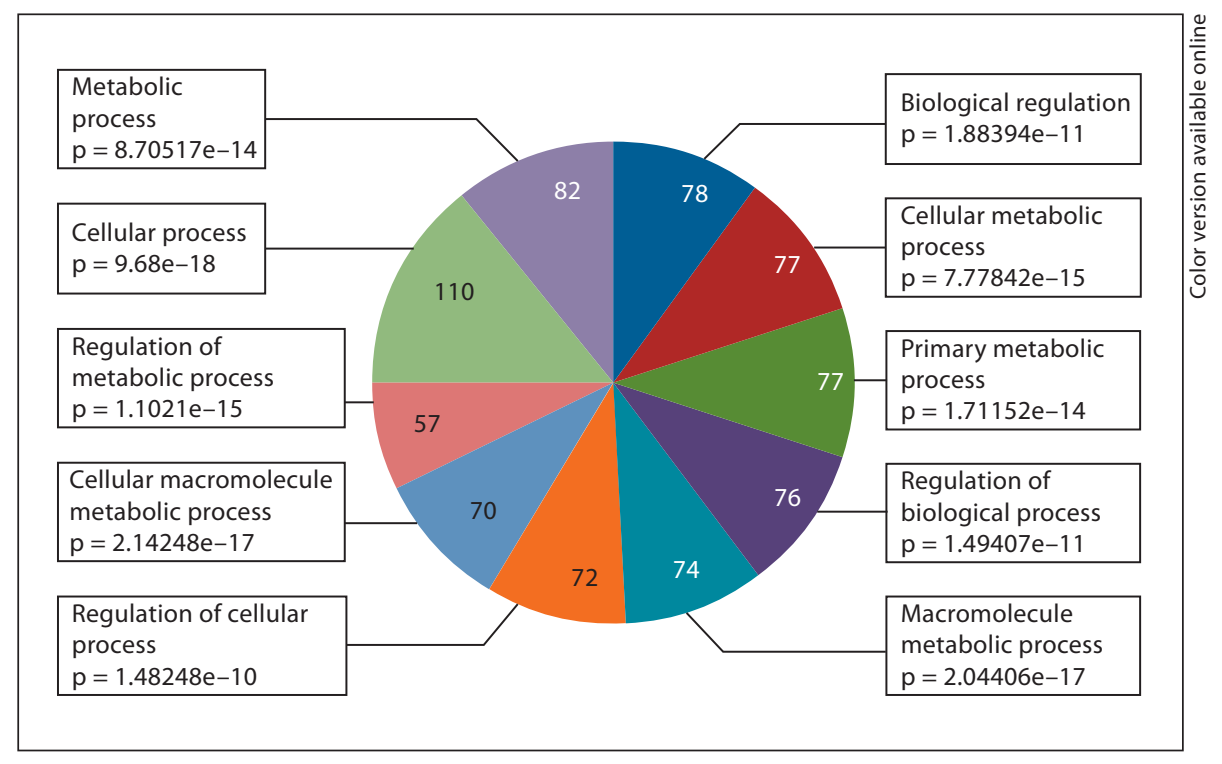

some cancers and degenerative diseases, which provides fresh impetus to the research of miRNAs in the pathogenesis of disease. miRNAs also play important roles during viral infection, and altered miRNA expression in response to viral infection could be associated with virulence [20-22]. However, the precise changes in miRNA expression involved in a host's response to CVB3 infection and the relationship between miRNA expression and pathogenesis remain largely unknown. Here we detected miRNA expression profiling in the target organ (heart) of mice infected with CVB3 on day 4 p.i. and analyzed the functions of the differentially expressed miRNAs.
In acute VMC, innate immunity plays key roles in the host defense against viral infection. CVB3 genome, the single-stranded RNA and double-stranded RNA replicating intermediates are potent pathogen-associated molecular patterns that induce pattern recognition receptor TLR3 [23-25], TLR7 [26-29], MDA5 [30], adaptor MyD88, and IRF7 expression upregulation 8-fold, 5-fold, 13-fold, 2-fold, and 61-fold, respectively. This upregulation resulted from the mRNA microarray analysis and CVB3 RNA to bind with these receptors to produce type II IFN, type I IFN, and proinflammatory cytokines for defending, eliminating the invading viruses, and causing apoptosis of virus-infected cells $[23,28,30]$. Thus, the in- 
nate anti-CVB3 immunity is synergistically mediated by TLR and RIG-I-like receptor, and type II IFN and type I IFN - two arms of innate immunity - exert their functions by coupling together to mount a full-blown antiviral response. As such, immune and inflammatory responses are stronger during the early infection phase.

In the present study, we detected and verified the inverse relationship between miR-146a expression upregulation and target TRAF6 and IRAK1 expression downregulation. Using a negative feedback mechanism, miR146a can regulate the inflammatory response to prevent lipopolysaccharide (LPS)-induced damage in THP-1 cells in a TLR-myeloid differentiation factor 88 (MyD88)-dependent manner by targeting TRAF6 and IRAK1 [31]. MiR-146a can also provide negative feedback in response to vesicular stomatitis virus infection-triggered type I IFN production in macrophages in an RIG-I-NF- $\kappa \mathrm{B}$-dependent manner by targeting TRAF6, IRAK1, and IRAK2, which could arouse virus replication increases [10]. For CVB3 infection, the TLR3-type III IFN axis is sufficient to reduce CVB3 replication and avoid this issue [23]. Thus, our results suggest that CVB3 infections induce miR-146a upregulation in vivo and that feedback of this negatively regulates immune and inflammatory responses, preventing inflammatory overactivation and returning the system to homeostasis.

Concurrently, miR-23a expression upregulation decreased the expression of the putative target CCL7, an important proinflammatory factor whose increasing levels can exacerbate myocardial inflammation [32, 33]. As such, miR-23a is probably another important negative regulator of VMC. That endogenous miRNAs directly downregulate proinflammatory factor expression may also be a feedback mechanism for hosts to regulate immune and inflammatory responses to preserve heart function and promote tissue repair. The mRNA microarray analysis also revealed that CXCL4 and CCL24 expressions were downregulated 2-fold and 3-fold, respectively.

We also detected miR-21 expression upregulation. MiR-21 can provide negative feedback that regulates the expression of $P D C D 4$, a proinflammatory protein that promotes activation of the transcription factor NF- $\kappa \mathrm{B}$ and suppresses IL-10; thus, miR-21 promotes IL-10 production upon LPS stimulation in vitro [34, 35]. In our study, we detected downregulation of the verified targets PDCD4 and IL12A and found 8-fold IL-10R upregulation using mRNA microarray analysis $[35,36]$. IL-10 is an anti-inflammatory cytokine that blocks NF- $\kappa \mathrm{B}$ and proinflammatory cytokines such as IL-12 [37], allowing the system to return to a homeostatic state. We did not find upregulated IL-10 gene expression using the mRNA microarray analysis or qPCR because the suppressive effect of PDCD4 on IL-10 occurred at the translational level, and increased eIF4E-directed translation can lead to increased mRNA turnover [38, 39]. As such, upon CVB3 infection, PDCD4 increasingly induced miR-21 upregulation, while feedback promoted IL-10 production. Therefore, miR-21 is another key miRNA resolution of inflammation in vivo.

The interaction between CVB3 and host cells can activate extracellular signal-regulated kinase and p38MAPK to enhance virus replication [40, 41]. Pharmacological inhibition of these MAPKs has been shown to greatly reduce CVB3 replication and viral progeny release in tissue culture cells and animal models [42]. In this study, we detected downregulated expression of MAP2K3, the putative target of miR-21. Using mRNA microarray analysis, we also found that MAP2K6, MAP4K3, and MAPKAPK3 expressions were downregulated 3-fold, 2-fold, and 2-fold, respectively. MAP2K3 and MAP2K6 phosphorylate and activate p38MAPK, while ERK and p38MAPK phosphorylate and activate MAPKAPK3. As such, decreases in MAPK2K3 and MAP2K6 resulted in decreased p38MAPK expression, which then reduced MAPKAPK expression. These results suggest that miRNAs could decrease cardiac viral load by inhibiting the MAPK signal pathway.

JAK-STAT signaling is activated and required for the early innate defense against CVB3 infection in the heart. SOCS family members have been observed to negatively regulate CVB3-induced JAK and STAT signaling with accompanying increases in viral replication, cardiomyopathy, and mortality in infected mice $[43,44]$. In the present study, we detected SOCS1 expression downregulation, the putative target of miR374. Using mRNA microarray analysis, we also found a 2 -fold downregulated SOCS5 expression, and an 8-fold, 4-fold, and 3-fold upregulated expression of STAT1, SATA2, and STAT5, respectively. As such, miRNAs can regulate the JAK-STAT pathway by inhibiting SOCS, which can augment the host-cell antiviral system, thus preventing viral-mediated heart damage during the early stages of infection.

In summary, this study provides miRNA expression profiling in heart tissue in the early phase of VMC in which several miRNAs were differentially expressed. We demonstrated the regulating roles of miRNAs in the pathogenesis of VMC by analyzing the function of partial target genes. Nevertheless, further in-depth studies are 
also being conducted, for example, the verification of additional target genes using luciferase reporter gene methods and dynamically observing miRNA expression levels in the heart. In this way, we can more clearly understand the pathogenesis of VMC in an effort to discover novel therapies for VMC.

\section{Acknowledgements}

We thank Xiao Liu for his help with data analysis. This work was partly supported by grants from the Beijing Natural Science Foundation (7102023). The findings and conclusions in this report are those of the authors and do not necessarily represent the views of the funding agency.

\section{References}

1 Kim DS, Nam JH: Characterization of attenuated coxsackievirus B3 strains and prospects of their application as live-attenuated vaccines. Expert Opin Biol Ther 2010;10: 179-190.

-2 Meister G, Tuschl T: Mechanisms of gene silencing by double-stranded RNA. Nature 2004;431:343-349.

-3 Bartel DP: MicroRNAs: genomics, biogenesis, mechanism and function. Cell 2004;116: 281-297.

4 Ambros V: The functions of animal microRNAs. Nature 2004;431:350-355.

5 Bartel DP: MicroRNAs: target recognition and regulatory functions. Cell 2009;136: 215-233.

-6 Lee R, Feinbaum R, Ambros V: A short history of a short RNA. Cell 2004;116:S89-S92.

7 Wienholds E, Kloosterman WP, Miska E, Alvarez-Saavedra E, Berezikov E, de Bruijn E, Horvitz HR, Kauppinen S, Plasterk RH: MicroRNA expression in zebrafish embryonic development. Science 2005;309:310-311.

-8 Jopling CL, Yi M, Lancaster AM, Lemon SM, Sarnow P: Modulation of hepatitis $C$ virus RNA abundance by a liver-specific microRNA. Science 2005;309:1577-1581.

$\checkmark 9$ Lecellier CH, Dunoyer P, Arar K, LehmannChe J, Eyquem S, Himber C, Voinnet OA: Cellular microRNA mediates antiviral defense in human cells. Science 2005;308:557560.

10 Hou J, Wang P, Lin L, Liu X, Ma F, An H, Wang Z, Cao X: MicroRNA-146a feedback inhibits RIG-I-dependent type I IFN production in macrophages by targeting TRAF6, IRAK1, and IRAK2. J Immunol 2009;183: 2150-2158.

-11 Li Y, Chan EY, Li J, Ni C, Peng X, Rosenzweig E, Tumpey TM, Katze MG: MicroRNA expression and virulence in pandemic influenza virus-infected mice. J Virol 2010;84: 3023-3032.

- 12 Bhomia M, Balakathiresan N, Sharma A, Gupta P, Biswas R, Maheshwari R: Analysis of microRNAs induced by Venezuelan equine encephalitis virus infection in mouse brain. Biochem Biophys Res Commun 2010; 395:11-16.
13 Zhao P, Zhao L, Zhang T, Wang H, Qin C, Yang S, Xia X: Changes in microRNA expression induced by rabies virus infection in mouse brains. Microb Pathog 2012;52:4754.

14 Liu Z, Carthy CM, Cheung P, Bohunek L, Wilson JE, McManus BM, Yang D: Structural and functional analysis of the $5^{\prime}$ untranslated region of coxsackievirus B3 RNA: in vivo translational and infectivity studies of full-length mutants. Virology 1999;265: 206-217.

$\checkmark 15$ Lewis BP, Shih IH, Jones-Rhoades MW, Bartel DP, Burge CB: Prediction of mammalian microRNA targets. Cell 2003;115:787-798.

16 John B, Sander C, Marks DS: Prediction of human microRNA targets. Methods $\mathrm{Mol}$ Biol 2006;342:101-113.

17 Wang X, El Naqa IM: Prediction of both conserved and nonconserved microRNA targets in animals. Bioinformatics 2008;24:325332.

18 Guo H, Ingolia NT, Weissman JS, Bartel DP: Mammalian microRNAs predominantly act to decrease target mRNA levels. Nature 2010; 466:835-840.

19 Huang JC, Babak T, Corson TW, Chua G, Khan S, Gallie BL, Hughes TR, Blencowe BJ, Frey BJ, Morris QD: Using expression profiling data to identify human microRNA targets. Nat Methods 2007;4:1045-1049.

20 Gottwein E, Cullen BR: Viral and cellular microRNAs as determinants of viral pathogenesis and immunity. Cell Host Microbe 2008;3:375-387.

21 Dykxhoorn DM: MicroRNAs in viral replication and pathogenesis. DNA Cell Biol 2007;26:239-249.

22 Cullen BR: Viral and cellular messenger RNA targets of viral microRNAs. Nature 2009;457:421-425.

23 Negishi H, Osawa T, Ogami K, Ouyang X, Sakaguchi S, Koshiba R, Yanai H, Seko Y, Shitara H, Bishop K, Yonekawa H, Tamura T, Kaisho T, Taya C, Taniguchi T, Honda K: A critical link between Toll-like receptor 3 and type II interferon signaling pathways in antiviral innate immunity. Proc Natl Acad Sci USA 2008;105:20446-20451.
24 Hardarson HS, Baker JS, Yang Z, Purevjav E, Huang CH, Alexopoulou L, Li N, Flavell RA, Bowles NE, Vallejo JG: Toll-like receptor 3 is an essential component of the innate stress response in virus-induced cardiac injury. Am J Physiol Heart Circ Physiol 2007; 292:H251-H258.

25 Richer MJ, Lavallée DJ, Shanina I, Horwitz MS: Toll-like receptor 3 signaling on macrophages is required for survival following coxsackievirus B4 infection. PLoS One 2009; 4:e4127.

26 Crozat K, Beutler B: TLR7: a new sensor of viral infection. Proc Natl Acad Sci USA 2004; 101:6835-6836.

27 Heil F, Hemmi H, Hochrein H, Ampenberger F, Kirschning C, Akira S, Lipford G, Wagner $\mathrm{H}$, Bauer S: Species-specific recognition of single-stranded RNA via toll-like receptor 7 and 8. Science 2004;303:1526-1529.

28 Wessely R, Klingel K, Knowlton KU, Kandolf $\mathrm{R}$ : Cardioselective infection with Coxsackievirus B3 requires intact type I interferon signaling. Circulation 2001;103:756-761.

29 Wang JP, Asher DR, Chan M, Kurt-Jones EA, Finberg RW: Antibody-mediated TLR7-dependent recognition of viral RNA. J Immunol 2007;178:3363-3367.

30 Wang JP, Cerny A, Asher DR, Kurt-Jones EA, Bronson RT, Finberg RW: MDA5 and MAVS mediate type I interferon responses to Coxsackie B virus. J Virol 2010;84:254-260.

- 31 Taganov KD, Boldin MP, Chang KJ, Baltimore D: NF-kappaB-dependent induction of microRNA miR-146, an inhibitor targeted to signaling proteins of innate immune responses. Proc Natl Acad Sci USA 2006;103: 12481-12486.

32 Michalec L, Choudhury BK, Postlethwait E, Wild JS, Alam R, Lett-Brown M, Sur S: CCL7 and CXCL10 orchestrate oxidative stress-induced neutrophilic lung inflammation. J Immunol 2002;168:846-852.

-33 Westermann D, Savvatis K, Lindner D, Zietsch C, Becher PM, Hammer E, Heimesaat MM, Bereswill S, Völker U, Escher F, Riad A, Plendl J, Klingel K, Poller W, Schultheiss HP, Tschöpe C: Reduced degradation of the chemokine MCP-3 by matrix metalloproteinase- 2 exacerbates myocardial inflammation in experimental viral cardiomyopathy. Circulation 2011;124:2082-2093. 
34 Hilliard A, Hilliard B, Zheng SJ, Sun H, Miwa T, Song W, Chen YH: Translational regulation of autoimmune inflammation and lymphoma genesis by programmed cell death 4. J Immunol 2006;177:8095-8102.

35 Sheedy FJ, Palsson-McDermott E, Hennessy EJ, Martin C, O'Leary JJ, Ruan Q, Johnson DS, Chen Y, O'Neill LA: Negative regulation of TLR4 via targeting of the proinflammatory tumor suppressor PDCD4 by the microRNA miR-21. Nat Immunol 2010;11:141147.

-36 Lu TX, Munitz A, Rothenberg ME: MicroRNA-21 is up-regulated in allergic airway inflammation and regulates IL-12p35 expression. J Immunol 2009;182:4994-5002.

37 Murray, PJ: STAT3-mediated anti-inflammatory signaling. Biochem Soc Trans 2006; 34:1028-1031.
38 Rousseau D, Kaspar R, Rosenwald I, Gehrke L, Sonenberg N: Translation initiation of ornithine decarboxylase and nucleocytoplasmic transport of cyclin D1 mRNA are increased in cells overexpressing eukaryotic initiation factor 4E. Proc Natl Acad Sci USA 1996;93:1065-1070.

39 Graff JR, De Benedetti A, Olson JW, Tamez P, Casero RA Jr, Zimmer SG: Translation of ODC mRNA and polyamine transport are suppressed in ras-transformed CREF cells by depleting translation initiation factor $4 \mathrm{E}$. Biochem Biophys Res Commun 1997;240: 15-20.

40 Luo H, Yanagawa B, Zhang J, Luo Z, Zhang M, Esfandiarei M, Carthy C, Wilson JE, Yang D, McManus BM: Coxsackievirus B3 replication is reduced by inhibition of the extracellular signal-regulated kinase (ERK) signaling pathway. J Virol 2002;76:3365-3373.

41 Si X, Luo H, Morgan A, Zhang J, Wong J, Yuan J, Esfandiarei M, Gao G, Cheung C, McManus BM: Stress-activated protein kinases are involved in coxsackievirus B3 viral progeny release. J Virol 2005;79:1387513881.
42 Marchant D, Dou Y, Luo H, Garmaroudi FS, McDonough JE, Si X, Walker E, Luo Z, Arner A, Hegele RG, Laher I, McManus BM: Bosentan enhances viral load via endothelin-1 receptor type-A-mediated p38 mitogenactivated protein kinase activation while improving cardiac function during coxsackievirus-induced myocarditis. Circ Res 2009;104:813-821.

43 Knowlton KU: Unsolved medical issues and new targets for further research in viral myocarditis and dilated cardiomyopathy. Ernst Schering Res Found Workshop 2006;55:1935.

44 Yasukawa H, Yajima T, Duplain H, Iwatate M, Kido M, Hoshijima M, Weitzman MD, Nakamura T, Woodard S, Xiong D, Yoshimura A, Chien KR, Knowlton KU: The suppressor of cytokine signaling-1 (SOCS1) is a novel therapeutic target for enterovirusinduced cardiac injury. J Clin Invest 2003; 111:469-478. 